\title{
MAXIMUM DEGREE AND FRACTIONAL MATCHINGS IN UNIFORM HYPERGRAPHS
}

\section{Zoltán FÜREDI}

\author{
Mathematical Institute of the \\ Hungarian Academy of Sciences \\ Budapest, Hungary $\mathrm{H}-1053$
}

Received 11 January 1980

Let $\mathscr{H}$ be a family of $r$-subsets of a finite set $X$. Set $D(\mathscr{H})=\max _{x \in X}|\{E: x \in E \in \mathscr{H}\}|$, (maximum degree). We say that $\mathscr{H}$ is intersecting if for any $H, H^{\prime} \in \mathscr{H}$ we have $H \cap H^{\prime} \neq \emptyset$. In this case, obviously, $D(\mathscr{H}) \geqq|\mathscr{H}| / r$. According to a well-known conjecture $D(\mathscr{H}) \geqq|\mathscr{H}| /(r-1+1 / r)$. We prove a slightly stronger result. Let $\mathscr{H}$ be an $r$-uniform, intersecting hypergraph. Then either it is a projective plane of order $r-1$, consequently $D(\mathscr{H})=|\mathscr{H}| /(r-1+1 / r)$, or $D(\mathscr{H}) \geqq|\mathscr{H}| /(r-1)$. This is a corollary to a more general theorem on not necessarily intersecting hypergraphs.

\section{Introduction, definitions}

\subsection{Some well-known definitions}

We list the basic definitions and notation to be used throughout: hypergraph $\mathscr{H}$ - a finite collection of non-empty finite sets (edges); vertex set of $\mathscr{H}-V(\mathscr{H})=\cup\{E: E \in \mathscr{H}\}$; rank of $\mathscr{H}-r(\mathscr{H})=\max \{|E|: E \in \mathscr{H}\}$,

$\mathscr{H}$ is $r$-uniform if the cardinality of every $E \in \mathscr{H}$ is $r$; degree of a vertex $x$ (in $\mathscr{H})-d_{\mathscr{H}}(x)=|\{E: x \in E \in \mathscr{H}\}|$; $D(\mathscr{H})=\max \left\{d_{\mathscr{H}}(x): x \in V(\mathscr{H})\right\}$

$\mathscr{H}$ is $D$-regular if the degree of every vertex $x$ is $D$; partial hypergraph - $\mathscr{H}^{\prime} \subset \mathscr{H}$

matching - partial hypergraph of $\mathscr{H}$ whose edges are pairwise disjoint; $v(\mathscr{H})$ - matching number - maximum number of edges in a matching; intersecting hypergraph $-v(\mathscr{H})=1$;

transversal (or cover) - a set $T \subset V(\mathscr{H})$ which meets all the edges;

$\tau(\mathscr{H})$ - transversal number - minimum cardinality of a transversal. 


\subsection{Fractional transversals and matchings}

A survey with applications on fractional hypergraph theory can be found e.g. in Berge [1] or Lovász [12], [16]. Here we shall define only the most important concepts of this theory which appeared in first papers published on this topic. (Berge and Simonovits [2], Lovász [11].) satisfying

A fractional transversal of a hypergraph $\mathscr{H}$ is a weight function $t: V(\mathscr{H}) \rightarrow \mathbf{R}$

and

$$
t(x) \geqq 0 \quad \text { for every } \quad x \in V(\mathscr{H}),
$$

$$
\sum_{x \in E} t(x) \geqq 1 \text { for every edge } E \in \mathscr{H} \text {. }
$$

The value of a fractional transversal $t$ is

$$
|t|=\sum_{x \in V(\mathscr{V})} t(x)
$$

The minimum of $|t|$ when $t$ ranges over all fractional transversals is called the fractional transversal number and is denoted by

$$
\tau^{*}(\mathscr{H})=\min \{|t|: t \text { is a fractional transversal of } \mathscr{H}\} .
$$

Similarly the fractional matching number is the maximum value of the fractional matchings of $\mathscr{H}$, i.e.

$v^{*}(\mathscr{H})=\max \left\{\sum_{E \in \mathscr{H}} w(E) \mid w: \mathscr{H} \rightarrow \mathbf{R}, w(E) \geqq 0, \forall x \in V(\mathscr{H})\right.$ we have $\left.\sum_{E \ni x} w(E)\right\} \leqq 1$.

Clearly, to determine the fractional transversal number and the fractional matching number is a problem of linear programming. This is a dual pair so by the duality principle of linear programming we have $\tau^{*}(\mathscr{H})=v^{*}(\mathscr{H})$ for every hypergraph $\mathscr{H}$. Thus

$$
1 \leqq \nu \leqq v^{*}=\tau^{*} \leqq \tau \leqq r v .
$$

In view of the fact that $w(E) \equiv 1 / D$ and $t(x) \equiv 1 / \mathrm{min}|E|$ are a fractional matching resp. fractional transversal we have

$$
\frac{|\mathscr{H}|}{D(\mathscr{H})} \leqq \tau^{*}(\mathscr{H}) \leqq \frac{|V(\mathscr{H})|}{\min \{|E|: E \in \mathscr{H}\}} .
$$

\subsection{An important example}

If $\mathscr{H}$ is $D$-regular and $r$-uniform then (1) yields

$$
|\mathscr{H}| / D=|V(\mathscr{H})| / r=\tau^{*}(\mathscr{H}) .
$$

For $r \geqq 3$ write $\mathscr{P}_{r}$ for the hypergraph consisting of the lines of the $r$-uniform finite projective plane (if there exists) further let $\mathscr{P}_{2}$ consist of the 2-tuples of a 3-element set (i.e. $\mathscr{P}_{2}$ is a triangle) and let $\mathscr{P}_{1}$ be the hypergraph having only 1 point. It is well-known that $\mathscr{P}_{r}$ exists provided $r=P+1$, where $P$ is a prime power.

It is evident that every line of the projective plane $\mathscr{P}_{r}$ is a minimal transversal of $\mathscr{P}_{r}$. For $r=1,2,3$ there is no other minimal transversal. For the projective 
plane $\mathscr{P}_{r}$ with $r>3$, J. Pelikán [18] proved that the only transversals of cardinality $r$ are the edges, and all other transversal sets have size $\geqq r+2$.

Summing up: $\left|\mathscr{P}_{r}\right|=\left|V\left(\mathscr{P}_{r}\right)\right|=r^{2}-r+1, \mathscr{P}_{r}$ is $r$-uniform and $r$-regular, $v\left(\mathscr{P}_{r}\right)=1$, $\tau\left(\mathscr{P}_{r}\right)=r, \tau^{*}\left(\mathscr{P}_{r}\right)=r-1+1 / r$.

\section{Results}

Considering all the $r$-tuples of an underlying set with $r v+r-1$ elements it can be seen that the inequality $\tau \leqq r \gamma$ cannot be improved in general. Nevertheless, as L. Lovász observed, the inequality $\tau^{*} \leqq r v$ is not sharp. He showed (see [14], [15]), that, for any hypergraph $\mathscr{H}, \tau^{*}(\mathscr{H})<r(\mathscr{H}) v(\mathscr{H})$, furthermore

$$
\tau^{*}(r, v)=\sup \left\{\tau^{*}(\mathscr{H}): r(\mathscr{H}) \leqq r, v(\mathscr{H}) \leqq v\right\}<r v .
$$

For $\nu=1$ he proved that $\tau^{*}(r, 1) \leqq r-1+2 /(r+1)$ and he conjectured that $\tau^{*}(r, 1) \leqq r-1+1 / r$. In this paper we shall prove a bit more.

Theorem. Let $\mathscr{H}$ be a hypergraph of rank $r \geqq 3, v(\mathscr{H})=v$. Suppose further tha ${ }^{t}$ $\mathscr{H}$ does not contain a partial hypergraph which consists of $p+1$ copies of pairwise disjoint r-uniform projective planes. Then

$$
\tau^{*}(\mathscr{H}) \leqq(r-1) v+p / r .
$$

(The proof of the Theorem is in $\S 5$.) We mention that the inequality of the Theorem is sharp. To see this consider the hypergraph $\mathscr{P}_{r}^{\prime}$ which we get from $\mathscr{P}_{r}$ by omitting a line. $\left(\tau^{*}\left(\mathscr{P}_{r}^{\prime}\right)=r-1\right.$. $)$

The case $r=1$ is of no importance. For $r=2$ the Theorem does not hold true, because for the odd circuits $C_{2 n+1}$ one has $v\left(C_{2 n+1}\right)=n, p=0$ but $\tau^{*}\left(C_{2 n+1}\right)$ $=n+1 / 2$. L. Lovász [13] proved that for an ordinary graph $G$

$$
\tau^{*}(G) \leqq \frac{1}{2}(\tau+v) \leqq \frac{3}{2} v .
$$

The following corollaries are true even if $r<3$.

Corollary 1. If $\mathscr{H}$ is the union of $v$ pairwise disjoint copies of $\mathscr{P}_{r}$, then $\tau^{*}(\mathscr{H})=$ $=(r-1+1 / r) v$ otherwise $\tau^{*}(\mathscr{H}) \leqq(r-1+1 / r) v-1 / r$.

Proof. The inequality $\tau^{*}(\mathscr{H})>(r-1+1 / r) v-1 / r$ implies that $\mathscr{H}$ has a partial hypergraph $\mathscr{H}_{v}$ which is the disjoint union of $v$ copies of $\mathscr{P}_{r}$. That is $\mathscr{H}_{v} \subset \mathscr{H}$. Then it follows from (3) that $\mathscr{H}_{v} \equiv \mathscr{H}$.

(The case $r=2$ is left to the reader).

Corollary 2. Let $r$ be a positive integer for which $\mathscr{P}_{r}$ does exist. Then $\tau^{*}(r, v)=$ $=(r-1+1 / r) v$. If $\mathscr{P}_{r}$ does not exist then $\tau^{*}(r, v) \leqq(r-1) v$.

I think that for the time being the determination of the exact value of $\tau^{*}(r, v)$ for other $r$ 's is hopelessly difficult, because to solve this problem one probably has to decide whether or not the projective plane $\mathscr{P}_{r}$ does exist for a given $r$. 


\section{Applications}

\subsection{The maximum degree of hypergraphs}

Let us consider an intersecting, $r$-uniform hypergraph $\mathscr{H}$. Obviously $D(\mathscr{H})$ $\geqq|\mathscr{H}| / r$. It is a well-known conjecture $[6,7]$ that $D \geqq|\mathscr{H}| /(r-1+1 / r)$. From the Theorem and from (1) a slightly stronger result follows.

Corollary 3. Let $\mathscr{H}$ be an r-uniform, intersecting hypergraph. Then either it is a projective plane of order $r-1$ and consequently $D(\mathscr{H})=|\mathscr{H}| /(r-1+1 / r)$ or $D(\mathscr{H})$ $\geqq|\mathscr{H}| /(r-1)$.

In general $D(\mathscr{H}) \geqq|\mathscr{H}| /(r-1+1 / r) v$. This was proved for $r=2$ by B. Bollobás [4] in a slightly different form. This Corollary is sharp because $D\left(\mathscr{P}_{r}^{\prime}\right)=\left|\mathscr{P}_{r}^{\prime}\right| /(r-1)$.

\subsection{The number of vertices of regular hypergraphs}

Using his result $\left(\tau^{*}(r, 1) \leqq r-1+2(r+1)\right)$ mentioned above L. Lovász [14], [15] proved the following conjecture of P. Erdős [6] and B. Bollobás [4]:

If $\mathscr{H}$ is an intersecting, $r$-uniform and regular hypergraph, then $|V(\mathscr{H})|$ $\leqq r^{2}-r+1$.

By the Theorem and (2) we generalize this result as follows. (For $r=2$ see Bollobás-Eldridge [5]).

Corollary 4. If $\mathscr{H}$ is $r$-uniform and regular then $|V(\mathscr{H})| \leqq\left(r^{2}-r+1\right) v$. Moreover equality holds if and only if $\mathscr{H}$ is the disjoint union of projective planes or order $r-1$. Furthermore if there is no such $r$-uniform plane then $|V(\mathscr{H})| \leqq\left(r^{2}-r\right) v$.

By omitting from $\mathscr{P}_{r}$ a point together with all the lines containing it we get the hypergraph $\mathscr{P}_{r}^{\prime \prime}$ which is $(r-1)$-regular, $r$-uniform and intersecting. It has $r^{2}-r$ points. This example shows that Corollary 4 is sharp, too.

\subsection{Fractional transversal number of r-partite hypergraphs}

The hypergraph $\mathscr{H}$ is said to be r-partite if $V(\mathscr{H})$ is the disjoint union of $X_{1}, \ldots, X_{r}$, and for each $E \in \mathscr{H}:\left|E \cap X_{i}\right|=1$ holds $(i=1,2, \ldots, r)$.

A well-known conjecture of $\mathrm{H}$. J. Ryser states that for an $r$-partite hypergraph $\tau \leqq(r-1) v$. (In particular for $r=2$ this is simply König's Theorem (see [12]). For some small values of $r$ and $v$ this conjecture has recently been proved by Zs. Tuza [19]). A. Gyárfás [10] proved an easier version of Ryser's conjecture. His result follows from our Theorem because $\mathscr{P}_{r}$ is not $r$-partite $(r \geqq 2)$.

Corollary 5. If the hypergraph $\mathscr{H}$ is $r$-partite then $\tau^{*}(\mathscr{H}) \leqq(r-1) v(\mathscr{H})$.

This Corollary is sharp for $\mathscr{P}_{r}^{\prime}$ is $r$-partite and $\tau^{*}\left(\mathscr{P}_{r}^{\prime}\right)=r-1$.

3.4 Some further applications of this Theorem to extremal graphs and to extremal set-systems can be found in J. Pach-L. Surányi [17], Z. Füredi [9] and P. Frankl-Z. Füredi [8], respectively. 


\section{The reduction lemma} value $M$.

We denote by $\left(\mathbf{a}_{i}, b_{i}, \mathbf{c}, I\right)$ the following linear program with minimum

$$
\left\{\begin{aligned}
\mathbf{x} & \geqq 0 \\
\mathbf{a}_{i} \mathbf{x} & \geqq b_{i} \quad \text { for all } i \in I . \\
\min \mathbf{c x} & =M\left(\mathbf{a}_{i}, b_{i}, \mathbf{c}, I\right)
\end{aligned}\right.
$$

Here $\mathbf{a}_{i}, \mathbf{x}$ and $\mathbf{c}$ are $n$-dimensional vectors, $b_{i}$ 's are real numbers and $|I|$ is the number of conditions of the program $(|I|<\infty)$.

The following Proposition is well known in the theory of linear programming. For the sake of completness we give its short proof in the Appendix.

Proposition. If the linear program $\left(\mathbf{a}_{i}, b_{i}, \mathbf{c}, I\right)$ with $n$ variables has a finite optimum, then there exists a $J \subset I$ such that $M\left(\mathbf{a}_{i}, b_{i}, \mathbf{c}, I\right)=M\left(\mathbf{a}_{i}, b_{i}, \mathbf{c}, J\right)$ and $|J| \leqq n$.

In other words this Proposition states that the number of conditions of a linear program can be reduced to $n$ without changing the optimum value.

The proof of the Theorem is based on the following Lemma which may help to determine $\tau^{*}$ in some other cases as well.

Lemma. For any hypergraph $\mathscr{H}$ there exists a partial hypergraph $\mathscr{H}^{\prime} \subset \mathscr{H}$ such that $\tau^{*}\left(\mathscr{H}^{\prime}\right)=\tau^{*}(\mathscr{H})$ and $\left|\mathscr{H}^{\prime}\right| \leqq\left|V\left(\mathscr{H}^{\prime}\right)\right|$.

Proof. To determine $\tau^{*}$ one has to solve a linear program of dimension $|V(\mathscr{H})|$, with index set $I$, where $|I|=|\mathscr{H}|$. Of course, this program always has a finite optimum. So by applying the Proposition (possibly several times) one can find a suitable $\mathscr{H}^{\prime} \subset \mathscr{H}$.

\section{Proof of the Theorem}

Let $\mathscr{H}$ be an $r$-uniform hypergraph which does not contain $p+1$ disjoint copies of the projective plane $\mathscr{P}_{r}$ and $v(\mathscr{H})=v$. Suppose $r \geqq 3$. (Our proof can be applied for $r=2$, too, but the details are left to the reader.)

It is sufficient to give a suitable fractional transversal $t$ of $\mathscr{H}$. We shall give it by induction on $v$, while $r$ is fixed. The proof in the case $v=1$ is similar to that one for $v>1$ and that is why we do not separate them, but sometimes we mention the differences.

For $\mathscr{H}=\emptyset$ put $\tau^{*}(\mathscr{H})=0$. By the Lemma we may suppose that $|\mathscr{H}|$ $\leqq|V(\mathscr{H})|$. Consequently,

$$
\min _{x \in V(\mathscr{H})} d(x) \leqq \frac{\sum\{d(x): x \in V(\mathscr{H})\}}{|V(\mathscr{H})|}=\frac{r|\mathscr{H}|}{|V(\mathscr{H})|} \leqq r .
$$

Case 1. There exists $x_{0} \in V(\mathscr{H})$ with $d_{\mathscr{H}}\left(x_{0}\right)=k<r$. Put $\mathscr{H}_{0}=\left\{E \in \mathscr{H}: x_{0} \in E\right\}$ $=\left\{E_{1}, \ldots, E_{k}\right\}$, and $\mathscr{H}_{i}=\left\{E \in \mathscr{H}: E \cap E_{i}=\emptyset\right\}$ for $1 \leqq i \leqq k$.

For the hypergraph $\mathscr{H}_{i}$ the induction hypothesis can be applied, because $v\left(\mathscr{H}_{i}\right) \leqq v-1$ and of course $\mathscr{H}_{i}$ does not contain more than $p$ disjoint $\mathscr{P}_{r}$ as partial 
hypergraphs. Hence there exists a fractional transversal $t_{i}: V\left(\mathscr{H}_{i}\right) \rightarrow \mathbf{R}$ of $\mathscr{H}_{i}$, such that $\left|t_{i}\right| \leqq(r-1)(v-1)+p / r$. (If here $v=1$ then $\mathscr{H}_{i}=\emptyset, t_{i} \equiv 0$.)

Put

$$
t(x)= \begin{cases}0 & \text { if } \quad x=x_{0} \\ \frac{1}{k}\left(d_{0}(x)+\sum_{i=1}^{k} t_{i}(x)\right) & \text { if } \quad x \in V(\mathscr{H})-\left\{x_{0}\right\},\end{cases}
$$

where $d_{0}(x)$ is the degree of $x$ in the hypergraph $\mathscr{H}_{0}$. We claim that this is a fractional transversal of $\mathscr{H}$. Indeed, $t(x) \geqq 0$, and for any $E \in \mathscr{H}_{0}$ we have

$$
\sum_{x \in E} t(x) \geqq \frac{1}{k}\left(\sum_{x \in E-\left\{x_{0}\right\}} d_{0}(x)\right) \geqq \frac{1}{k}(r-1) \geqq 1 .
$$

If $E \in \mathscr{H}-\mathscr{H}_{0}$ then

Finally

$$
\begin{aligned}
\sum_{x \in E} t(x) & =\frac{1}{k}\left(\sum_{x \in E} d_{0}(x)+\sum_{i=1}^{k}\left[\sum_{x \in E} t_{i}(x)\right]\right) \\
& \geqq \frac{1}{k}\left(\sum_{\substack{i=1 \\
E \cap E_{i} \neq \varnothing}}^{k} 1+\sum_{\substack{i=1 \\
E \cap E_{i}=\varnothing}}^{k} 1\right)=1 .
\end{aligned}
$$

$$
\begin{aligned}
\tau^{*}(\mathscr{H}) & \leqq|t|=\frac{1}{k}\left(\sum_{x \in V(\mathscr{H})-\left\{x_{0}\right)} d_{0}(x)+\sum_{i=1}^{k}\left[\sum_{x \in V(\mathscr{H})} t_{i}(x)\right]\right) \\
& =\frac{1}{k}\left((r-1) k+\sum_{i=1}^{k}\left|t_{i}\right|\right) \leqq(r-1) v+p / r .
\end{aligned}
$$

(For $v=1$ we get $\tau^{*}(\mathscr{H}) \leqq r-1$.)

Case 2. $\min _{x \in V(\mathscr{H})} d_{\mathscr{H}}(x) \geqq r$.

Then, by (5), $\mathscr{H}$ is $r$-regular, so $|\mathscr{H}|=|V(\mathscr{H})|$. We shall show that $|\mathscr{H}|$ $\leqq\left(r^{2}-r\right) v+p$ from here, by (2), the Theorem follows.

Suppose on the contrary that $|\mathscr{H}| \geqq\left(r^{2}-r\right) v+p+1$. Let $E_{1}$ be an arbitrary edge of $\mathscr{H}$ and put $\mathscr{H}_{1}=\left\{E \in \mathscr{H}: E \cap E_{1}=\emptyset\right\}$. Applying the induction hypothesis to $\mathscr{H}_{1}$ and using (1) we get that

$$
|\mathscr{H}|=\left|\left\{E: E \cap E_{1} \neq \emptyset\right\}\right|+\left|\mathscr{H}_{1}\right| \leqq 1+r(r-1)+\tau^{*}\left(\mathscr{H}_{1}\right) D\left(\mathscr{H}_{1}\right) \leqq\left(r^{2}-r\right) v+p+1 .
$$

Here the right side is at most $\left(r^{2}-r\right) v+p$ if there is an edge $E$ with $\left|E \cap E_{1}\right| \geqq 2$. Consequently it is enough to consider the following case.

$$
\begin{aligned}
& |\mathscr{H}|=|V(\mathscr{H})|=v\left(r^{2}-r\right)+p+1 \\
& \left|\mathscr{H}-\mathscr{H}_{1}\right|=r^{2}-r+1
\end{aligned}
$$

(If $v=1$ and $p=1$ then (6) yields a contradiction, because in this case $\mathscr{H}_{1}=\emptyset$. Similarly, for $v=1, p=0$ we have by (6) and (7) that $\mathscr{H}$ is an $r$-uniform, $r$-regular, intersecting system of sets on $\left(r^{2}-r+1\right)$ points with the same number of edges. 
This in turn implies that $\mathscr{H} \equiv \mathscr{P}_{r}$ contradicting to $p=0$ From now on we suppose that $v \geqq 2$.)

Let $E_{1}, E_{2}, \ldots, E_{v}$ any fixed matching. We call an edge $E \in \mathscr{H}$ crossing if it intersects more than one $E_{i}(1 \leqq i \leqq v)$. We count the number of edges of $\mathscr{H}-\left\{E_{1} \ldots E_{v}\right\}$ with multiplicities in the poins of $\bigcup_{i=1}^{v} E_{i}$. We get $v r(r-1)$. From the other hand $\left|\mathscr{H}-\left\{E_{1}, \ldots, E_{v}\right\}\right|=v\left(r^{2}-r\right)+p+1-v$, and so there are at most $v-1-p$ crossing edges. This means that there is an edge, say $E_{1}$, in the matching $\left\{E_{1} \ldots E_{v}\right\}$ which intersects at most one crossing edge.

Case $2 a . E_{1}$ is not intersected by a crossing edge.

In other words, the system $\left\{E_{2}, \ldots, E_{v}\right\}$ has no common point with edges intersecting $E_{1}$. Since $v(\mathscr{H})=v$ we get that $\mathscr{H}-\mathscr{H}_{1}$ is an intersecting family. Further $D\left(\mathscr{H}-\mathscr{H}_{1}\right)=r$, and this together with (6) and (7) implies that $\mathscr{H}-\mathscr{H}_{1} \equiv_{\mathscr{P}_{r}}$. Moreover, the underlying sets $V\left(\mathscr{H}_{1}\right)$ and $V\left(\mathscr{H}-\mathscr{H}_{1}\right)$ are disjoint. Applying the induction hypothesis to $\mathscr{H}_{1}$ with parameters $v-1$ and $p-1$ we get by (1)

$$
\left|\mathscr{H}-\mathscr{H}_{1}\right| \leqq \tau^{*}\left(\mathscr{H}-\mathscr{H}_{1}\right) r \leqq\left(r^{2}-r\right)(v-1)+(p-1),
$$

contradicting to (6).

Case $2 b$. There is a unique crossing edge $E^{\prime}$ intersecting $E_{1}$.

It remains true that $\mathscr{H}-\mathscr{H}_{1}-\left\{E^{\prime}\right\}$ is an intersecting family, $\left|\mathscr{H}_{-}-\mathscr{H}_{1}-\left\{E^{\prime}\right\}\right|=$ $=r^{2}-r, D\left(\mathscr{H}-\mathscr{H}_{1}-\left\{E^{\prime}\right\} \mid=r\right.$. We claim that in this case $\mathscr{H}-\mathscr{H}_{1}-\left\{E^{\prime}\right\} \equiv \mathscr{P}_{r}^{\prime}$. Indeed, by (7), every edge of $\mathscr{H}-\mathscr{H}_{1}-\left\{E^{\prime}\right\}$ contains a point of degree $r-1$. There are exactly $r$ points of this type, they form a set $T$. It is easy to check that $\left(\mathscr{H}-\mathscr{H}_{1}-\left\{E^{\prime}\right\}\right) \cup\{T\}$ is a finite projective plane. $E^{\prime} \neq T$ for $E^{\prime}$ is crossing. So there is an edge $E^{\prime \prime} \in \mathscr{H}-\mathscr{H}_{1}$ such that $E^{\prime} \cap E^{\prime \prime}=\emptyset$, and

(8) at least $r-1$ edges $E$ of $\mathscr{H}-\mathscr{H}_{1}$ have the property that $E \cap E^{\prime} \neq \emptyset$ and $E \cap E^{\prime \prime} \neq \emptyset$.

Let $\mathscr{H}^{1}=\left\{E \in \mathscr{H}: E \cap E^{\prime}=\emptyset\right.$ and $\left.E^{\prime \prime} \cap E=\emptyset\right\}$ and $\mathscr{H}^{2}=\mathscr{H}-\mathscr{H}^{1}$. Applying the induction hypothesis to $\mathscr{H}^{1}$ with parameters $v-2$ and $p$, and using that upper bound for $\left|\mathscr{H}^{2}\right|$ which follows from (8), we get

$$
|\mathscr{H}|=\left|\mathscr{H}^{1}\right|+\left|\mathscr{H}^{2}\right| \leqq(v-2)\left(r^{2}-r\right)+p+2\left(r^{2}-r\right)+2-(r-1) .
$$

This again contradicts to (6), provided $r \geqq 3$.

\section{Appendix: Proof of the Proposition}

Dropping some of the inequalities of (4) the minimal value of the program can only decrease. Hence we have to prove that there is a $J \subset I,|J|=n$ such that $M\left(\mathbf{a}_{i}, b_{i}, \mathbf{c}, J\right) \geqq M\left(\mathbf{a}_{i}, b_{i}, \mathbf{c}, I\right)=M$.

Suppose, on the contrary, that for every $J \subset I,|J|=n$, we have $M\left(\mathbf{a}_{i}, b_{i}, \mathbf{c}, J\right)<M$. This means that any $n$ of the halfspaces $\left\{\mathbf{y}: \mathbf{a}_{i} y \geqq b_{i}\right\}$ have a point in common with the open convex polytope $\left\{\mathbf{x} \in \mathbf{R}^{n}: \mathbf{c x}<M, \mathbf{x} \geqq 0\right\}$. The system (4) has a solution, hence any $n+1$ of the halfspaces $\left\{\mathbf{y}: \mathbf{a}_{i} \mathbf{y} \geqq b_{i}\right\}$ have a point 
in common. Now Helly's Theorem in $\mathbf{R}^{n}$ implies that the intersection of the $|I|+1$ convex sets $\left\{\mathbf{y}: \mathbf{a}_{i} \mathbf{y} \geqq b_{i}\right\}$ and $\{\mathbf{x}: \mathbf{c x}<M, \mathbf{x} \geqq 0\}$ is not empty, i.e. it contains a point $\mathbf{x}_{0}$. This point $\mathbf{x}_{0}$ is feasible for the program (4) and $\mathbf{c x}_{0}<M=M\left(\mathbf{a}_{i}, b_{i}, \mathbf{c}, I\right)$. This contradiction proves the existence of the appropriate $J$.

Acknowledgment. I would like to express my thanks to P. Frankl and I. Bárány for their help.

\section{References}

[1] C. Berge, Packing problems and hypergraph theory: a survey, Annals of Discrete Math. 4. (1979), 3-37.

[2] C. Berge and M. Simonovits, The coloring number of the direct product of two hypergraphs, in [3], 21-33.

[3] C. Berge and D. K. RAY-Chaudhuri eds., Hypergraph Seminar 1972, Lecture Notes 411 (Springer-Verlag, Berlin, 1972).

[4] B. BollobÁs, Disjoint triples in a 3-graph with given maximal degree, Quart, J. Math. Oxford, Sec. Ser. 28 (1977), $81-87$.

[5] B. Bollobás and S. E. Eldridge, Maximal matchings in graphs with given minimal and maximal degrees, Math. Proc. Cambridge Phil. Soc. 79 (1976), 221-234.

[6] P. ERDós, private communication.

[7] P. FrankL, On intersecting families of finite sets, J. Combinatorial Th. (A) 24 (1978), 146-161.

[8] P. FRANKL and Z. Füredi, Disjoint $r$-tuples in an $r$-graph with given maximal degree, submitted to Quart, J. Math. Oxford.

[9] Z. FÜREDI, Erdős-Ko-Rado type theorems with upper bounds on the maximal degree, in: Algebraic Methods in Graph Theory, (Proc. Conf. Szeged 1978, L. Lovász et al. eds.) Coll. Math. Soc. J. Bolyai 25, North-Holland 1981, to appear.

[10] A. GÝ́RFÁs, to appear.

[11] L. LovÁsz, Normal hypergraphs and the perfect graph conjecture, Discrete Math. 2 (1972), $253-267$.

[12] L. Lovász, Combinatorial problems and exercises, Akadémiai Kiadó, Budapest-North-Holland, Amsterdam 1979.

[13] L. LovÁsz, Minimax theorems for hypergraphs, in [3], 111-126.

[14] L. LovÁsz, Doctoral thesis, Szeged 1977.

[15] L. LovÁsz, On minimax theorems of combinatorics (in Hungarian) Matematikai Lapok 26 (1975), 209-264.

[16] L. LovÁsz, Graph theory and integer programming, Annals of Discrete Math., 2 (1978)

[17] J. PACH and L. SURÁNYI, Graphs of diameter 2 and linear programming, in Algebraic Methods in Graph Theory, (Proc. Conf. Szeged 1978, L. Lovász et al. eds.) Coll. Math. Soc. J. Bolyai 25, North-Holland 1981, to appear.

[18] J. Pelikén, Properties of balanced incomplete block designs, in: Combinatorial Theory and its Applications (P. Erdős, A. Rényi and V. T. Sós, eds.) Coll. Math. Soc. J. Bolyai 4, NorthHolland 1971), 869-890.

[19] Zs. TuzA, Some special cases of Ryser's conjecture, to appear. 